\title{
An Easily Accessible Cathode Buffer Layer for Achieving Multiple High Performance Polymer Photovoltaic Cells
}

Wenchao Zhao ${ }^{\dagger}$, Long Ye $e^{+, *}$, Shaoqing Zhang, Huifeng $\mathrm{Yao}^{+}$, Mingliang Sun ${ }^{\dagger, *}$ and Jianhui Hou ${ }^{t, *}$

${ }^{\dagger}$ Institute of Material Science and Engineering, Ocean University of China, Qingdao 266100, P. R. China. E-mail: mlsun@ouc.edu.cn

${ }^{*}$ State Key Laboratory of Polymer Physics and Chemistry, Beijing National Laboratory for Molecular Sciences, Institute of Chemistry, Chinese Academy of Sciences, Beijing 100190, P. R. China. E-mail: yelong@iccas.ac.cn, hjhzlz@iccas.ac.cn, Tel: +86-10-82615900.

\section{Contents of Supporting Information}

S1. The inverted PBDT-TS1:PC ${ }_{71} B M-b a s e d ~ P S C s$ devices with different temperature annealed $\mathrm{Ba}(\mathrm{OH})_{2}$ as the CBLs.

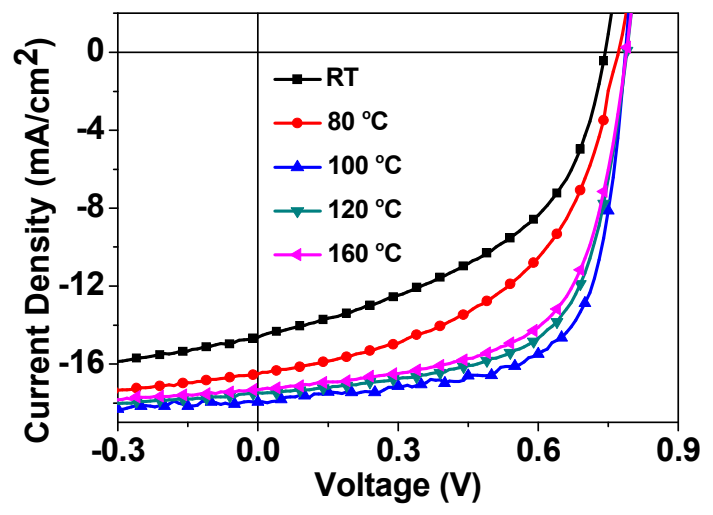


Fig. S1 $J-V$ characteristics of the inverted PBDT-TS1:PC ${ }_{71} \mathrm{BM}$-based PSC devices with different temperature annealed $\mathrm{Ba}(\mathrm{OH})_{2}$ as the CBLs.

Table S1 The photovoltaic performance of the PBDT-TS1:PC ${ }_{71} \mathrm{BM}$-based PSCs with different temperature annealed $\mathrm{Ba}(\mathrm{OH})_{2}$ as the CBLs under the illumination of AM $1.5 \mathrm{G}, 100 \mathrm{~mW} / \mathrm{cm}^{2}$.

\begin{tabular}{ccccc}
\hline Temperature $\left({ }^{\circ} \mathrm{C}\right)$ & $\boldsymbol{V}_{\boldsymbol{c o c}}(\mathbf{V})$ & $\boldsymbol{J}_{\boldsymbol{s c}}\left(\mathbf{m A} / \mathbf{c m}^{2}\right)$ & $\boldsymbol{F F}(\%)$ & $\boldsymbol{P C E}(\%)$ \\
\hline $\mathrm{RT}$ & 0.743 & 14.63 & 47.39 & 5.15 \\
80 & 0.771 & 16.51 & 50.58 & 6.44 \\
100 & 0.787 & 17.94 & 67.42 & 9.52 \\
120 & 0.790 & 17.48 & 64.30 & 8.88 \\
160 & 0.789 & 17.32 & 62.16 & 8.49 \\
\hline
\end{tabular}

S2. The PBDT-TS1:PC ${ }_{71} \mathrm{BM}-\mathrm{based}$ device with different concentration of $\mathrm{Ba}(\mathrm{OH})_{2}$ as the CBLs

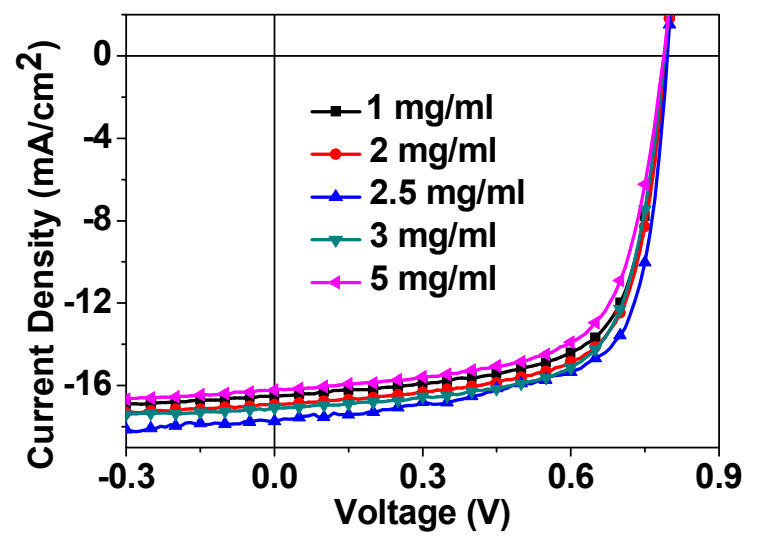

Fig. S2 $J-V$ characteristics of the PBDT-TS1:PC ${ }_{71} \mathrm{BM}$-based conventional PSCs with different concentration of $\mathrm{Ba}(\mathrm{OH})_{2}$ as the CBLs. 
Table S2 The photovoltaic performance of the PBDT-TS1:PC ${ }_{71} \mathrm{BM}$-based PSCs with different concentration of $\mathrm{Ba}(\mathrm{OH})_{2}$ as the CBLs.

\begin{tabular}{ccccc}
\hline $\begin{array}{c}\text { Concentration } \\
(\mathrm{mg} / \mathrm{ml})\end{array}$ & $V_{o c}(\mathrm{~V})$ & $J_{s c}\left(\mathrm{~mA} / \mathbf{c m}^{2}\right)$ & $F F(\%)$ & $P C E(\%)$ \\
1 & 0.792 & 16.50 & 67.96 & 8.88 \\
2 & 0.793 & 16.93 & 68.44 & 9.19 \\
2.5 & $\mathbf{0 . 7 9 6}$ & $\mathbf{1 7 . 7 3}$ & $\mathbf{6 8 . 3 9}$ & $\mathbf{9 . 6 5}$ \\
3 & 0.790 & 17.09 & 68.63 & 9.27 \\
5 & 0.788 & 16.22 & 66.44 & 8.49 \\
\hline
\end{tabular}

S3. The surface morphologies of the active layer (PBDT-TS1:PC ${ }_{71} B M$ ) with and without a $\mathrm{Ba}(\mathrm{OH})_{2} \mathrm{CBL}$.

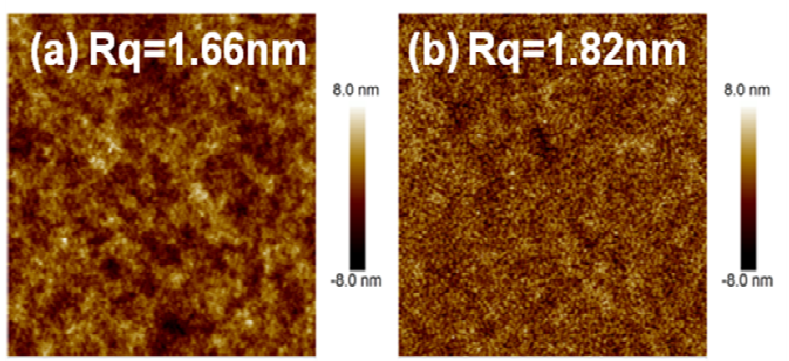

Fig. S3 AFM height images of the active layers without a CBL (a) and with a $\mathrm{Ba}(\mathrm{OH})_{2}$ buffer layer (b).

\section{Complete References}

[26] Guo, X. G.; Zhou, N. J.; Lou, S. J.; Smith, J.; Tice, D. B.; Hennek, J. W.; Ortiz,

R. P.; Navarrete, J. T. L.; Li, S. Y.; Strzalka, J.; Chen, L. X.; Chang, R. P. H.; 
Facchetti, A.; Marks, T. J. Polymer Solar Cells with Enhanced Fill Factors. Nat. Photonics 2013, 7, 825-833.

[31] You, J. B.; Chen, C. C.; Dou, L. T.; Murase, S.; Duan, H. S.; Hawks, S. A.; Xu, T.; Son, H. J.; Yu, L. P.; Li, G.; Yang, Y. Metal Oxide Nanoparticles as an Electron-Transport Layer in High-Performance and Stable Inverted Polymer Solar Cells. Adv. Mater. 2012, 24, 5267-5272.

[33] Nguyen, T. L.; Choi, H.; Ko, S. J.; Uddin, M. A.; Walker, B.; Yum, S.; Jeong, J. E.; Yun, M. H.; Shin, T. J.; Hwang, S.; Kim, J. Y.; Woo, H. Y. Semi-Crystalline Photovoltaic Polymers with Efficiency Exceeding 9\% in a Similar to $300 \mathrm{~nm}$ Thick Conventional Single-Cell Device. Energy Environ. Sci. 2014, 7, 3040-3051.

[57] Zhou, N. J.; Lin, H.; Lou, S. J.; Yu, X. G.; Guo, P. J.; Manley, E. F.; Loser, S.; Hartnett, P.; Huang, H.; Wasielewski, M. R.; Chen, L. X.; Chang, R. P. H.; Facchetti, A.; Marks, T. J. Morphology-Performance Relationships in High-Efficiency All-Polymer Solar Cells. Adv. Energy Mater. 2014, 4, 1300785.

[59] Zhou, Y.; Kurosawa, T.; Ma, W.; Guo, Y. K.; Fang, L.; Vandewal, K.; Diao, Y.; Wang, C. G.; Yan, Q. F.; Reinspach, J.; Mei, J. G.; Appleton, A. L.; Koleilat, G. I.; Gao, Y. L.; Mannsfeld, S. C. B.; Salleo, A.; Ade, H.; Zhao, D. H.; Bao, Z. N. High Performance All-Polymer Solar Cell via Polymer Side-Chain Engineering. $A d v$. Mater. 2014, 26, 3767-3772.

[75] Mu, C.; Liu, P.; Ma, W.; Jiang, K.; Zhao, J. B.; Zhang, K.; Chen, Z. H.; Wei, Z. H.; Yi, Y.; Wang, J. N.; Yang, S. H.; Huang, F.; Facchetti, A.; Ade, H.; Yan, H. 
High-Efficiency All-Polymer Solar Cells Based on a Pair of Crystalline Low-Bandgap Polymers. Adv. Mater. 2014, 26, 7224-7230. 